\title{
Rückwärts in die Zukunft?
}

Die Urabstimmung der FMH verlangt die Unterstützung des Referendums gegen die Managed-Care-Vorlage, die eben in den Schlussabstimmungen der beiden Parlamentskammern mit grossem Mehr angenommen wurde. Aber die Zukunft hat bereits begonnen ...

Felix Huber

Facharzt für Allgemeine Medizin, Medizinischer Leiter mediX zürich
Korrespondenz:

Dr. med. Felix Huber Rotbuchstrasse 46

CH-8037 Zürich

Tel. 0443653030

Fax 0443653040

felix.huber@medix.ch
Bereits heute haben $51 \%$ der Versicherten in der Schweiz ein Hausarzt- oder ein verwandtes Modell. Damit hat sich der Anteil seit 2007 mehr als verdoppelt. 54\% der Haus- und Kinderärzte in der Schweiz sind in einem Hausarztmodell tätig. Dieser Trend wird sich rasant fortsetzen. Warum brauchen wir dann diese KVG-Revision?

Mit der neuen Managed-Care-Regelung werden diese 51\% Versicherten neben den bereits heute tieferen Prämien (10-20\%) von einem tieferen Selbstbehalt profitieren. Sie bezahlen noch 500 statt wie bisher 700 Franken jährlich. Wer seine Behandlung nicht durch einen Arzt in einem Qualitätsnetz koordinieren lassen will, wird etwas mehr bezahlen - 300 Franken jährlich. Das sollte einem eine potentielle Überversorgung doch wert sein.

\section{Vorlage verbessert koordinierende integrierte Versorgung}

Integrierte Versorgung beruht auf einem Vertrag zwischen Ärztenetz und Kasse. Damit verschwinden die Listenmodelle, die von Kassen einseitig und ohne Vergütung für uns Ärzte propagiert werden. Der Vertrag wird freiwillig ausgehandelt. Vertragsvorschläge der Kassen kann man zurückweisen.

Der Risikoausgleich wird weiter verbessert. Das ist eine längst fällige Voraussetzung für ein Fairplay im Gesundheitswesen. Risikoselektion wird für die Kassen unattraktiv.

Die Budgetmitverantwortung erlaubt es den Ärztenetzen, ihre qualitativen und organisatorischen Anstrengungen finanzieren zu lassen. mediX zürich und bern mit je 100 Ärzten haben nie eine Mitgliedschaftsgebühr erhoben, in den meisten Jahren aber eine Überschussbeteiligung an die Partnerärzte ausbezahlt. Auch andere bekannte Ärztenetze, die seit langem mit Budgetmitverantwortungsverträgen arbeiten, mussten nie Beiträge von ihren Partnerärzten einfordern (Wintimed, Säntimed, Lumed). Diese Netze haben ihre grossen Anstrengungen für Qualitätsarbeit und Netzwerkentwicklung und -organisation immer über diese Budgetverträge finanziert. Budgetmitverantwortung führt zu seriöser Netzwerkarbeit. Das Risiko für das Ärztenetz ist minimal. Wir können das allen Netzen nur empfehlen.

Keine kasseneigenen Netze und Praxen mehr Das war eine wichtige Forderung der FMH und der Hausärzte Schweiz. Mit dem neuen Gesetz dürfen die Kassen keine eigenen Praxen und Netze mehr betreiben.
Fast mantraartig reklamieren die Gegner der Vorlage die Abschaffung der freien Arztwahl. Mitnichten. Die Versicherten haben immer noch die Möglichkeit, ihren Hausarzt im Netzwerk frei zu wählen. Mit einem Aufpreis von 300 Franken jährlich kann man sich unkoordiniert von Spezialist zu Spezialist bewegen. Aber das will bereits heute nur noch eine Minderheit der Versicherten.

Der Zugang zum Spezialisten und zum Spital wird vom Hausarzt zusammen mit dem Patienten geregelt. Viele Spezialisten sind bereits selber in Ärztenetzen und haben damit beste Erfahrungen gemacht. Viele Spezialisten sind auf «Preferred provider-Listen» der Ärztenetze, weil die Qualität und der Preis stimmen. Warum denn diese Angst vor Bevormundung durch die Ärztenetze?

Managed Care oder integrierte Versorgung ist bereits heute ein Mehrheitsmodell, sowohl bei den Versicherten wie bei den Haus- und Kinderärzten. Die neue Vorlage macht diese Netzwerke noch etwas attraktiver. Vor allem aber bringt sie mit der Budgetmitverantwortung eine willkommene Ernsthaftigkeit für eine optimale Koordination der medizinischen Behandlung und eine willkommene Finanzierung der Netzwerkaktivitäten durch die Kassen.

Gegen diese Vorlage zu sein, heisst rückwärts gewandt in die Zukunft zu blicken. Mit einem grossen Effort hat das Parlament eine ausgewogene Vorlage verabschiedet. Das ist eine konstruktive Zukunftslösung. Wird die Vorlage in der Referendumsabstimmung abgelehnt, dann wird sich die integrierte Versorgung ungebremst, aber etwas chaotisch und ziemlich heterogen weiterentwickeln. Die Kassen werden weiter unaufhaltsam Arztpraxen aufkaufen und Ärzte anstellen und mit der Zeit auch die medizinische Betreuungsphilosophie vorgeben. Es wird weiterhin Schlaumeierkassen wie die EGK und die Assura geben, die uns Ärzte einfach auf ein Listenmodell setzen und uns nichts bezahlen für unsere Qualitätsarbeit. Wollen wir das? Wird die Vorlage abgelehnt, wird auch der verbesserte Risikoausgleich auf sich warten lassen.

Die SP hat sich gegen die Vorlage entschieden, weil sie ihre Einheitskassen durchbringen will. Wollen wir denn noch mehr Regulierung durch das BAG? Glaubt jemand im Ernst, dass die eidgenössische Verwaltung uns bessere Arbeitsbedingungen schaffen wird?

Es wäre eine vornehme Haltung der Ärzteschaft, wenn sie die Zeichen der Zeit sehen und Hand bieten würde für eine konstruktive Verbesserung unserer ambulanten Versorgung. 